\title{
Transplantation of Telocytes Attenuates Unilateral Ureter Obstruction-Induced Renal Fibrosis in Rats
}

\author{
Long Zhenga,b Long $\mathrm{Li}^{\mathrm{e}}$ Guisheng $\mathrm{Qi}^{\mathrm{a}}$ Mushuang Hu $\mathrm{Hu}^{\mathrm{a}}$ Chao Hu $\mathrm{H}^{\mathrm{b}}$ Shuo Wang \\ Jiawei Lib Mingnan Zhang ${ }^{b} \quad$ Weitao Zhang ${ }^{a} \quad$ Yigang Zeng $^{b} \quad$ Yi Zhang ${ }^{b}$ \\ Liping Lib Xuanchuan Wang ${ }^{a}$ Miao Lin ${ }^{c}$ Tongyu Zhu ${ }^{a, b}$ Ruiming Ronga,d \\ 'Department of Urology, Zhongshan Hospital, Fudan University, Shanghai, ${ }^{b} S h a n g h a i$ Key Laboratory \\ of Organ Transplantation, 180 Fenglin Road, Shanghai, 'Department of Thoracic Surgery, Zhongshan \\ Hospital, Fudan University, Shanghai, 'Department of Blood Transfusion, Zhongshan Hospital, Fudan \\ University, Shanghai, eDepartment of Urology, Ninth People's Hospital, Shanghai Jiao Tong University \\ School of Medicine, Shanghai, China
}

\author{
Key Words \\ Telocyte • Renal fibrosis $• \mathrm{UUO} \cdot \mathrm{HGF}$
}

\begin{abstract}
Background/Aims: Previous studies imply that telocytes may have a protective effect on fibrosis in various organs, including the liver, colon, and heart. The effect of telocytes on renal fibrosis remains unknown. Herein, this study was designed to investigate the effect of telocytes on renal fibrosis and the potential mechanisms involved. Methods: In a unilateral ureteral obstruction (UUO)-induced renal fibrosis model, telocytes were injected via the tail vein every other day for 10 days. The degree of renal damage and fibrosis was determined using histological assessment. The expression of collagen I, fibronectin, epithelial-mesenchymal transition markers, and Smad2/3 phosphorylation was examined by western blot analyses. Real-time PCR and enzyme-linked immunosorbent assay were performed in vivo to detect the levels of transforming growth factor (TGF)- $\beta 1$ and various growth factors. Results: Telocytes attenuated renal fibrosis, as evidenced by reduced interstitial collagen accumulation, decreased expression of fibronectin and collagen I, upregulation of E-cadherin, and downregulation of $\alpha$-smooth muscle actin. Furthermore, telocytes decreased serum TGF- $\beta 1$ levels, suppressed Smad2/3 phosphorylation, and increased the expression of hepatocyte growth factor (HGF) in rat kidney tissue following UUO. Blockage of HGF counteracted the protective effect of telocytes on UUO-treated kidneys. Through the detection of HGF mRNA levels in vitro, we found that telocytes had no effect on HGF expression compared with renal fibroblasts. Conclusion: Telocytes attenuated UUO-induced renal fibrosis in rats, likely through enhancing the expression of HGF in an indirect manner.

L. Zheng, L. Li and G. Qi contributed equally to this work.

Miao Lin, Tongyu Zhu

and Ruiming Rong

Department of Urology, Zhongshan Hospital, Fudan University, Shanghai,

Department of Thoracic Surgery, Zhongshan Hospital, Fudan University, Shanghai

(China); Tel. 86-135 0168 2943, E-Mail rongruiming1969@163.com
\end{abstract}




\section{Introduction}

Renal fibrosis is considered the major cause of end-stage renal failure. Pathologically, it is characterized by hyperplasia of myofibroblasts and excessive deposition of extracellular matrix (ECM) [1-3]. ECM is mainly derived from myofibroblasts in the kidney. Excessive accumulation of ECM decreases the glomerular infiltration rate, deteriorates renal function, and results in eventual renal failure [4,5]. Renal fibrosis is a progressive process with a role in multiple clinical entities, including allograft injury, proteinuria, urinary tract obstruction, and diabetes mellitus [6, 7]. However, few strategies can effectively prevent or reverse renal fibrosis. Hence, the development of novel therapies that enable the prevention or attenuation of renal fibrosis is of urgent need.

Telocytes are acknowledged as a distinct type of interstitial cell identified recently in diverse organs, including the heart, lung, esophagus, pancreas, duodenum, jejunum, and kidney [8-16]. Multiple methods exist to distinguish telocytes from other types of cells. Telocytes share a specific small cell body and cellular prolongations referred to as telopodes, which are morphologically composed of a thin segment (podomer) and a thick segment (podom) [16]. Telocytes also co-express specific markers, including CD34, vimentin, CD117, and platelet-derived growth factor receptor $\alpha$ (PDGFR $\alpha$ ) [16]. Telocytes from different organs may display slight differences in markers. A previous study indicated that cardiac telocytes express either CD34, CD117, or PDGFR $\alpha$, while CD117 is not expressed in the telocytes of the gastrointestinal tract $[17,18]$. There is evidence that renal telocytes coexpress CD34, vimentin, and CD117 [16]. Furthermore, previous studies also demonstrated an obvious difference between telocytes and other cell populations, especially fibroblasts, in their microRNA imprint, proteome genetic profiles, and secretome levels [19-21]. These results indicate that telocytes are a unique cell population.

Telocytes are thought to be able to contribute to tissue generation. Li et al. found that telocyte transplantation decreased serum creatinine and blood urea nitrogen, attenuating renal dysfunction in a rat ischemia reperfusion injury model. Moreover, Li et al. found that this protective effect may be mediated by the secretion of paracrine growth factors [22]. Recently, the relationship between telocytes and fibrosis has received wide attention. Telocytes are decreased in number in systemic sclerosis, ulcerative colitis, Crohn's diseases, heart failure, and liver fibrosis [23-27], while the degree of their decrease correlated with the severity of fibrosis. Zhao et al. discovered that transplantation of telocytes decreased infarction size and improved myocardial function in myocardial infarctions, due to increased angiogenesis, decreased fibrosis at the infarct site and border zone, and improved pathological reconstruction of the left ventricle [26], suggesting that the transplantation of telocytes inhibited fibrosis. However, the effect of telocytes on renal fibrosis remains unknown.

In this study, we investigated the effect of telocytes on unilateral ureter obstruction (UUO)-induced renal fibrosis and the possible mechanisms involved.

\section{Materials and Methods}

\section{Experimental animals}

Thirty male Sprague-Dawley rats weighing 200-220 g were obtained from the Shanghai SLAC Lab. Animal Co., and bred in a specific pathogen-free grade experimental animal room. The rats had free access to food and water and were housed for 1 week before the experiments with a 12-h light-dark cycle. All animal procedures were performed in accordance with bioethics guidelines and were approved by the Bioethics Committee of Zhongshan Hospital, Fudan University, Shanghai, China.

\section{Cell culture}

The isolation of renal telocytes and primary culture were performed as described previously [22]. Briefly, rat kidney was obtained after a peritoneal heparin injection and perfusion of collagenase type II (Sigma-Aldrich, St. Louis, MI, USA). As the kidney softened, the renal capsule was incised and sterile samples 
were obtained and cut into pieces of approximately $1 \mathrm{~mm}^{3}$. Subsequently, the samples were digested with $10 \mathrm{mg} / \mathrm{mL}$ collagenase type II and $2000 \mathrm{U} / \mathrm{mL}$ deoxyribonuclease I (Sigma-Aldrich). Dispersed cells were collected and separated by filtration through a 40- $\mu$ m diameter cell strainer (BD Falcon, San Jose, CA, USA). Then, the collected cells were cultured with Dulbecco's modified Eagle's medium (DMEM)/F12 (Shanghai BioSun Sci\&Tech Co., Ltd., Shanghai, China). After 2-4 h of culture, renal glomerulus and tubule cells were removed. At $24 \mathrm{~h}$ later, fibroblast-like cells were scratched and removed under a phase contrast microscope (Olympus, Tokyo, Japan) and the cells with prolongations were kept.

Renal fibroblasts (NRK-49F) were purchased from the Aiyan Biological Research Company (Shanghai, China) and maintained in high-glucose DMEM (Shanghai BioSun Sci\&Tech Co., Ltd.). The rat renal tubular epithelial cell line NRK-52E was purchased from the Shanghai Branch of the Chinese Academy of Science (Shanghai, China) and cultured in DMEM/F12. NRK-49F and telocytes were seeded into Petri dishes ( $\mathrm{n}=3$ each), each dish containing $1.0 \times 10^{6}$ cells. After culture for 3 days, RNA was harvested from the NRK-49F cells and telocytes and used for real-time PCR analysis.

Transwell (Corning, Corning, NY, USA) was used for the co-culture experiment. The surface plates were seeded with NRK-49F cells or telocytes ( $\mathrm{n}=3$ each), and the ground plates were all seeded with NRK-52E cells. We added $100 \mu \mathrm{L}$ transforming growth factor (TGF)- $\beta 1$ (3 ng/mL; PeproTech, Inc., Rocky Hill, NJ, USA) to the ground plates. After co-culture for 3 days, RNA was extracted from the NRK-49F cells and telocytes and used for real-time PCR analysis.

The cells were divided randomly into 4 groups: control group, TGF- $\beta 1$ group, telocyte group, and fibroblast group. In the control and TGF- $\beta 1$ groups, $1.0 \times 10^{5} \mathrm{NRK}-52 \mathrm{E}$ cells plus $100 \mu \mathrm{L}$ phosphate-buffered saline (PBS) or TGF- $\beta 1$ were added to a 6-well plate; in the telocyte and fibroblast groups, a 6-well Transwell was used. The surface plates were seeded with NRK- $49 \mathrm{~F}$ cells or telocytes, and the ground plates were all seeded with NRK-52E cells. We added $100 \mu \mathrm{L}$ TGF- $\beta 1$ to the ground plates. After co-culture for 3 days, protein was harvested from the NRK-52E cells and used for western blot analysis.

\section{UUO-induced renal fibrosis model}

The rats were allocated randomly into six groups: sham group, UUO group, fibroblast group, telocyte group, anti-hepatocyte growth factor (HGF) antibody group, and IgG group ( $\mathrm{n}=5$ each). A UUO-induced renal fibrosis model was produced as described previously [28]. Briefly, the rats were anesthetized with pentobarbital $(40 \mathrm{mg} / \mathrm{kg})$. Then, the abdominal cavity was exposed, the left ureter was identified and ligated at two points with non-absorbable 5-0 silk sutures, and was cut between the ligatures. We injected 1 $\mathrm{mL}$ normal saline before closing the abdomen. The rats in the sham group underwent the same procedure, during which their ureters were manipulated but not ligated. In the fibroblast and telocyte groups, the rats were subjected to the same operation and $1.0 \times 10^{6}$ fibroblasts or telocytes were injected, respectively, via the tail vein every other day for 10 days. In the anti-HGF antibody and IgG groups, $5 \mathrm{mg} / \mathrm{kg}$ anti-HGF antibody (Santa Cruz Biotechnology, Santa Cruz, CA, USA) or IgG (Cell Signaling Technology, Inc., Danvers, MA, USA) was injected, respectively, via the tail vein every 5 days.

\section{Hematoxylin and eosin (H\&E) staining}

Paraffin-embedded rat tissues were cut into 5 - $\mu \mathrm{m}$ sections and subsequently deparaffinized and rehydrated before H\&E staining. The severity of renal interstitial injury was evaluated under light microscopy at 200× magnification. Tissue sections from the sham, UUO, fibroblast, and telocyte groups were evaluated for tubular dilation, interstitial expansion, and inflammatory infiltrate on a scale of 0-3: 0, normal tubulointerstitium; 1 , mild ( $\leq 25 \%$ ); 2 , moderate ( $>25 \%$ to $50 \%$ ); and 3, severe ( $>50 \%$ ), according to Lin $e t$ al [29].. Ten fields on each specimen were selected randomly and examined blindly by two examiners. The mean values of these estimations were used in the analyses.

\section{Masson staining}

After deparaffinization and rehydration, the sections were stained with Weigert's iron hematoxylin at room temperature for 5-10 min. The samples were then washed in water, polarized in acidic ethanol, and stained in Ponceau for 5-10 min. The slides were subsequently washed in phosphomolybdic acid and stained in Aniline blue. Ten fields of each specimen were chosen randomly and analyzed using Image-Pro Plus software 6.0 (Media Cybernetics, Rockville, MD, USA) to evaluate the degree of renal fibrosis.

\section{KARGER}




\section{Cellular Physiology Cell Physiol Biochem 2018;46:2056-2071

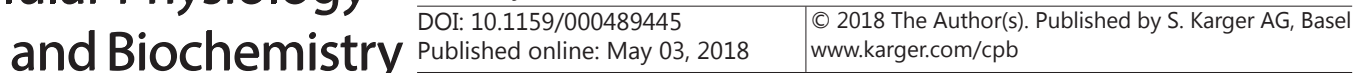 \\ Zheng et al.: Telocytes Attenuates Renal Fibrosis}

\section{Sirius red staining}

After being deparaffinized and rehydrated, the sections were incubated with a $0.1 \%$ Sirius red solution at room temperature for $1 \mathrm{~h}$. The slides were then washed twice in acidified water, dehydrated thrice in absolute alcohol, and cleared in xylene. Ten fields of each specimen were chosen randomly and analyzed using Image-Pro Plus software 6.0 (Media Cybernetics) to evaluate the degree of renal fibrosis.

\section{Immunofluorescence staining}

After purification, isolated renal telocytes and NRK-49F cells were fixed on a coverslip by using icecold methanol for $20 \mathrm{~min}$, and then incubated with 5\% serum for $30 \mathrm{~min}$ at room temperature. The cells were later incubated with primary antibodies overnight, including anti-CD34 (Abcam, Cambridge, UK), antiCD117 (Abcam), and anti-vimentin (EPIT MICS, Hangzhou, China) antibodies. Next, Alexa-donkey (Boster Biological Technology Co., Ltd., Wuhan, China) or FITC-goat anti-rabbit IgG (Jackson, West Grove, PA, USA) was added and incubated at room temperature for $1 \mathrm{~h}$. The cells were lastly counter-stained using DAPI.

The cells were examined by double immunofluorescence staining for CD34 and CD117. After being blocked with 5\% serum, anti-CD34 and anti-CD117 antibodies were added and incubated at $4^{\circ} \mathrm{C}$ overnight. Next, Alexa-donkey anti-rat IgG was added and incubated at room temperature for $1 \mathrm{~h}$. Subsequently, FITCgoat anti-rabbit IgG was added and the cells were incubated for $1 \mathrm{~h}$. The cells were lastly counter-stained using DAPI.

After deparaffinization and rehydration, the sections were washed with PBS. An anti-rabbit $\alpha$-smooth muscle actin (SMA) antibody (Abcam) was added and incubated at $4^{\circ} \mathrm{C}$ overnight. Next, Cy3-goat antirabbit IgG (Boster Biological Technology Co., Ltd.) was added and incubated at room temperature for $1 \mathrm{~h}$. Subsequently, an anti-mouse E-cadherin antibody (Boster Biological Technology Co., Ltd.) was added and incubated at $4^{\circ} \mathrm{C}$ for $12 \mathrm{~h}$, followed by FITC-goat anti-mouse IgG (BA1101; Boster Biological Technology Co., Ltd.) for $1 \mathrm{~h}$. Lastly, the sections were counter-stained by using DAPI.

\section{Western blot analysis}

Twenty micrograms protein was extracted from each kidney tissue sample, separated via 10\% sodium dodecyl sulfate-polyacrylamide gel electrophoresis, and transferred onto a polyvinylidene difluoride membrane. Primary antibodies, including anti-collagen-I (Abcam), anti-fibronectin (Abcam), anti-Ecadherin (Cell Signaling Technology), anti- $\alpha$-SMA (Cell Signaling Technology), anti-GAPDH (Cell Signaling Technology), anti-p-Smad2 (Cell Signaling Technology), anti-p-Smad3 (Cell Signaling Technology), antiSmad2/3 (Cell Signaling Technology), anti-ZEB1 (Cell Signaling Technology), anti-Snail (Cell Signaling Technology), and anti-HGF (Abcam) were incubated with the membrane at $4^{\circ} \mathrm{C}$ overnight under gentle shaking. After thorough washing, a horseradish peroxidase-conjugated goat anti-rabbit secondary antibody (Abcam) was added at room temperature for $1 \mathrm{~h}$ and visualized using an enhanced chemiluminescence system (Thermo Fisher Scientific, Rockford, IL, USA). Band intensity was analyzed by ImageJ and normalized to the value of GAPDH.

Enzyme-linked immunosorbent assay (ELISA) for serum TGF- $\beta 1$

TGF- $\beta 1$ levels in the serum samples were measured using a commercially available ELISA kit (Shanghai Xitang Biotechnology Co., Ltd., Shanghai, China) according to the manufacturer's instructions.

\section{Quantitative real-time PCR}

Total RNA was extracted from kidney tissue using the TRIzol reagent (Invitrogen, Carlsbad, CA). Four micrograms RNA was reversely transcribed into cDNA using a RevertAid First Strand cDNA Synthesis Kit (Thermo Fisher Scientific, Inc.) in accordance with the manufacturer's instructions. Real-time PCR was performed using a Mastercycler ep realplex system (Eppendorf, Hamburg, Germany). The thermocycling conditions were as follows: incubation for $2 \mathrm{~min}$ at $50^{\circ} \mathrm{C}$ and $10 \mathrm{~min}$ at $95^{\circ} \mathrm{C}$, which was then followed by a 2-step PCR program of $95^{\circ} \mathrm{C}$ for $15 \mathrm{~s}$ and $60^{\circ} \mathrm{C}$ for $20 \mathrm{~s}$ for 45 cycles. The levels of target genes were analyzed by reference to the value of GAPDH using the $2^{-\Delta \Delta \mathrm{Ct}}$ method. Gene-specific primers for rat fibronectin, collagen I, TGF- $\beta 1$, HGF, PDGF, BMP7, EGF, IGF-1, HO-1, and GAPDH were synthesized according to sequences listed in Table 1.

\section{Statistical analysis}

Data are expressed as the mean \pm standard deviation (SD). SPSS 19.0 software (SPSS Inc., Armonk, NY, USA) was used for statistical analysis of data among three groups with one-way analysis of variance 


\section{Cellular Physiology Cell Physiol Biochem 2018;46:2056-2071

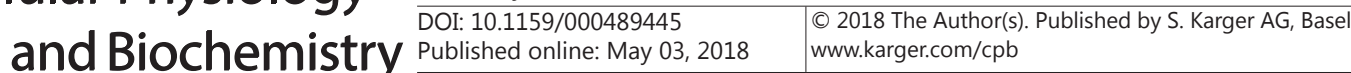 \\ Zheng et al.: Telocytes Attenuates Renal Fibrosis}

followed by Bonferroni's correction. $\mathrm{P}<0.05$ was considered statistically significant.

\section{Results}

Identification of renal telocytes

As shown in Fig. 1A, the primary culture of isolated telocytes was positive for CD34, CD117, and vimentin. Their prolongations also displayed alternating thick and thin segments. In contrast, fibroblasts were positive for vimentin, while negative for CD34 and CD117 (Fig. 1B). The purity of the isolated renal telocytes was determined by double immunofluorescence staining of CD34 and CD117. As shown in Fig. 1C, $96.36 \pm 2.06$ of the total cells were CD117-positive, while $95.00 \pm$ 2.68 were CD34-positive and $94.36 \pm 2.20$ were both CD34- and CD117-positive.

\section{Transplantation of telocytes attenuates UUO-induced renal injury and collagen deposition}

At 10 days after UUO, H\&E staining was
Table 1. Primers sequences used for RT-PCR

\begin{tabular}{ll}
\hline Gene & \multicolumn{1}{c}{ Primers } \\
\hline Collagen I & F: 5'-GAGAGAGCATGACCGATGGA-3' \\
& R: 5'-CGTGCTGTAGGTGAATCGAC-3' \\
Fibronectin & F: 5'-TGGAGAGACAGGAGGAAATAGC-3' \\
& R: 5'-CAGTGACAGCATACAGGGTGAT-3' \\
TGF-31 & F: 5'-CTTTGTACAACAGCACCCGC-3' \\
& R: 5'-CGGGTGACTTCTTTGGCGTA-3' \\
PDGF & F: 5'-AGTCGAGTCGGAAAGCTCAT-3' \\
& R: 5'-ACACCTCTGTACGCGTCTTG-3' \\
EGF & F: 5'-TGATTGAAATGGCCGATCTA-3' \\
& R: 5'-AACCACACGTGATCCTCAAA-3' \\
BMP-7 & F: 5'-ACAAGGCCGTCTTCAGTACC-3' \\
& R: 5'-CGCTCCCGGATGTAGTCC-3' \\
HGF & F: 5'-TATTTACGGCTGGGGCTACA-3' \\
& R: 5'-CGACCAGGAACAATGACAC-3' \\
IGF-1 & F: 5'-GGTGGACGCTCTTCAGTTCG-3' \\
& R: 5'-AGTACATCTCCAGCCTCCTCAG-3' \\
HO-1 & F: 5'-CAGAAGGGTCAGGTGTC-3' \\
& R: 5'-AGTAACTCCCACCTCGT-3' \\
GAPDH & F: 5'-AGGTCGGTGTGAACGGATTT-3' \\
& R: 5'-GGGGTCGTTGATGGCAACA-3' \\
\hline
\end{tabular}

performed in all groups to assess the degree of renal injury. As shown in Fig. 2, tubular structures had normal morphology and were neatly arranged in the sham group, while severe tubular dilation, interstitial expansion, and inflammatory cell infiltration were observed in the UUO group (for all online suppl. material, see www.karger.com/doi/10.1159/000489445, Suppl. Fig. 1). In contrast, transplantation of telocytes effectively attenuated tubulointerstitial lesions, while transplantation of fibroblasts led to no obvious change compared with the UUO group.

Sirius red and Masson staining were performed to detect collagen deposition. Compared to the sham group, there was a marked increase in collagen accumulation in the interstitium of kidney tissue in both the UUO and fibroblast groups, while transplantation of telocytes led to significantly less interstitial collagen deposition, though such results may not be comparable with the sham group. Besides, urine was collected from sham, UUO, fibroblast and telocyte groups, and urinary proteinuria was detected, but no significant difference was observed among groups (see online suppl. material, Suppl. Fig. 2), which may be due to the fact that the ureter was ligated into two points, and was cut between the ligatures, blocking urine containing proteinuria from the bladder.

Transplantation of telocytes reduces the deposition of ECM in kidney tissues

Fibronectin and collagen I are major components of ECM. As shown in Fig. 3A, the mRNA levels of fibronectin and collagen I were significantly increased in the UUO group compared with the sham group. Telocyte transplantation significantly decreased the levels of these indicators, whereas transplantation of fibroblasts did not lead to a significant change compared to the UUO group; these results were further confirmed by western blot. As shown in Fig. 3B, the expression of fibronectin and collagen I was obviously higher in both the UUO and fibroblast groups in comparison with the sham group, but telocyte treatment produced significantly lower expression of these indicators, though such results may not be comparable to the sham group, suggesting that the transplantation of telocytes reduced the deposition of ECM. 


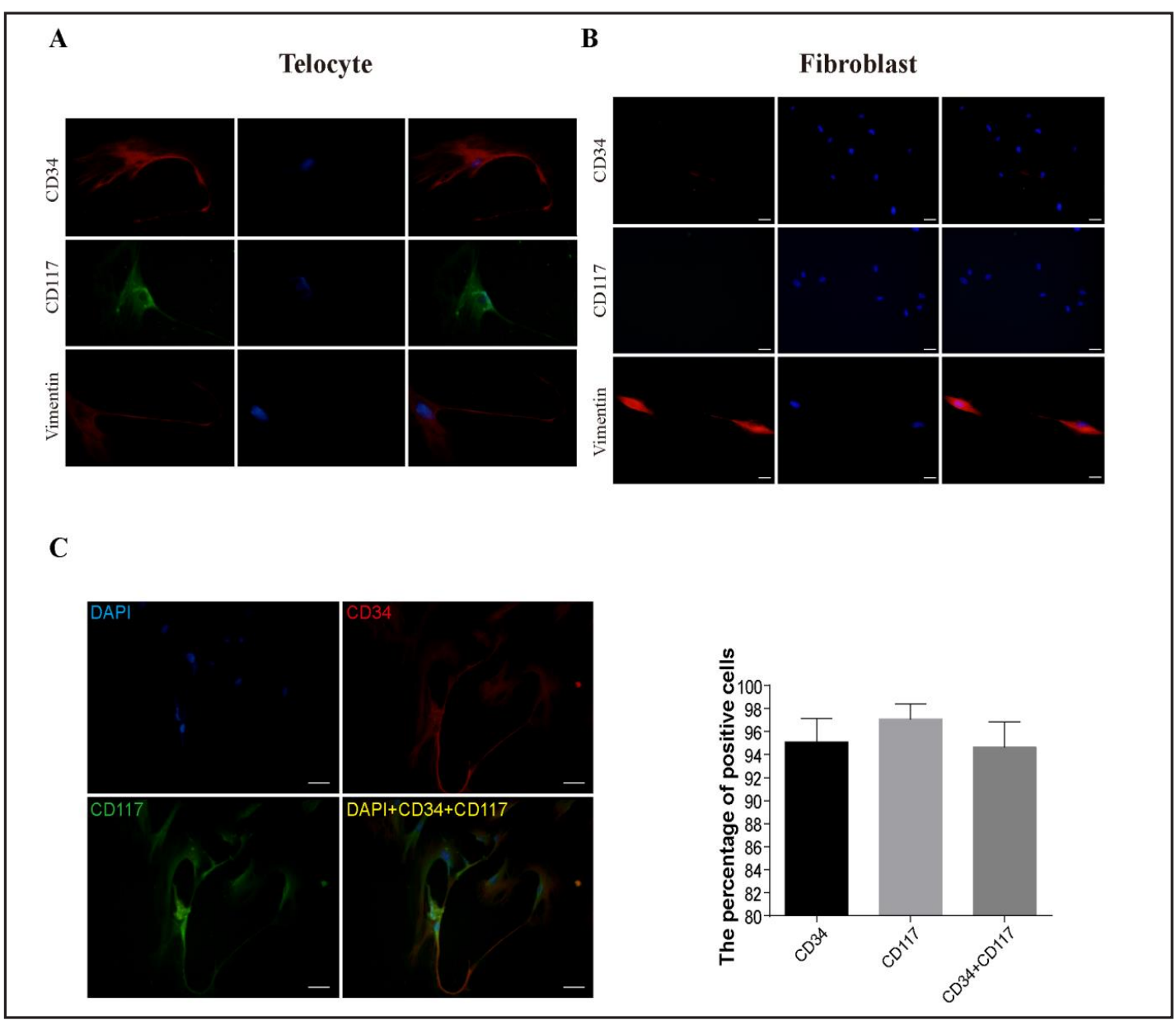

Fig. 1. Identification of renal telocytes. (A) Immunofluorescence labeling for CD34, CD117, and vimentin in telocytes. (B) Immunofluorescence labeling for CD34, CD117, and vimentin in fibroblasts (400x). (C) Double immunofluorescence staining against CD34 and CD117. Quantification of cells positive for CD34 and CD117, respectively, as well as both CD34 and CD117 (200×).

Telocyte transplantation inhibits epithelial-mesenchymal transition (EMT) in kidney tissue

EMT is the process underlying the change of epithelial cells to mesenchymal cells, which plays vital roles in the progression of renal fibrosis. The epithelial marker E-cadherin and the mesenchymal marker $\alpha$-SMA were examined to assess EMT. As shown in Fig. 3C, western blot analysis revealed the slight expression of $\alpha$-SMA in the sham group, while its expression was increased at 10 days after UUO. In contrast, the enhanced expression of E-cadherin was observed in the sham group and was significantly lower in the UUO group, indicating renal fibrosis. However, compared with the UUO group, telocyte transplantation led to a significant decrease of $\alpha$-SMA expression and increase of E-cadherin expression, while fibroblast transplantation showed no such effects. Similar results were also obtained from immunofluorescence staining (Fig. 4). These findings suggest that renal fibrosis occurred after UUO, as evidenced by the downregulation of E-cadherin and upregulation of $\alpha$-SMA. Transplantation of telocytes, but not fibroblasts, could partially reverse the change in the expression of these proteins, indicating its effect on the inhibition of EMT in kidney tissue.

Telocytes treatment decreases TGF- $\beta 1$ expression and its downstream signaling pathway in kidney tissue

TGF- $\beta 1$ is considered one of the most important regulators of renal fibrosis. To explore whether the attenuation of renal fibrosis by telocytes involved TGF- $\beta 1$, its levels were 


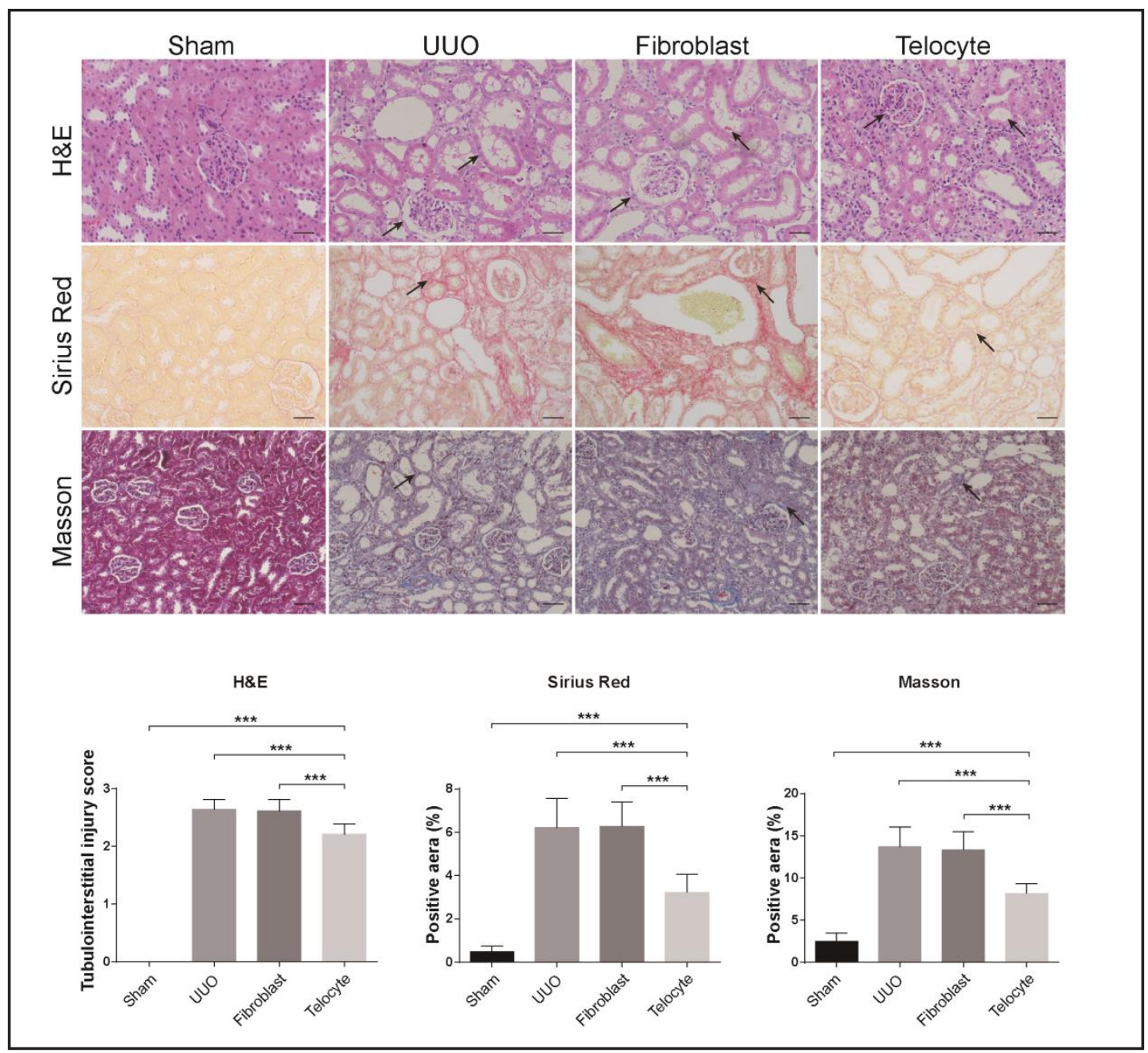

Fig. 2. Transplantation of telocytes attenuates UUO-induced renal injury and collagen deposition. Representative images of H\&E, Sirius red, and Masson staining in the sham, UUO, fibroblast, and telocyte groups. Quantification of tubular injury scores and tubulointerstitial collagen areas. Data are expressed as the mean $\pm \mathrm{SD}$. ${ }^{* * *} \mathrm{P}<0.001$.

examined in both serum and kidney tissue. As shown in Fig. 5A, the mRNA levels of TGF- $\beta 1$ were significantly elevated in the UUO group compared with the sham group, yet telocyte transplantation treatment seemed to decrease its levels, while transplantation of fibroblasts exhibited no such effect. TGF- $\beta 1$ levels in serum were also significantly increased in the UUO and fibroblast groups compared to the sham group. However, though higher than those of the sham group, TGF- $\beta 1$ levels were significantly lower in the telocyte group than in the UUO and fibroblast groups. These results revealed that telocyte treatment decreased TGF- $\beta 1$ expression.

To investigate further whether the TGF- $\beta 1$ signaling pathway is affected by telocyte treatment, western blot analysis was performed to detect the phosphorylation of Smad2/3. As shown in Fig. 5B, Smad2 and Smad3 phosphorylation was barely detectable in the sham group, yet it was significantly higher at 10 days after UUO. Telocyte treatment significantly blunted the increased phosphorylation of Smad2 and Smad3 brought about by UUO, while treatment with fibroblasts displayed no such effects. Snail and ZEB1 are target genes regulated by the Smad complex. Western blot analysis revealed the obviously higher expression of Snail and ZEB1 in both the UUO and fibroblast groups compared with the sham group. Telocyte treatment led to the decreased expression of Snail and ZEB1, suggesting

\section{KARGER}


A
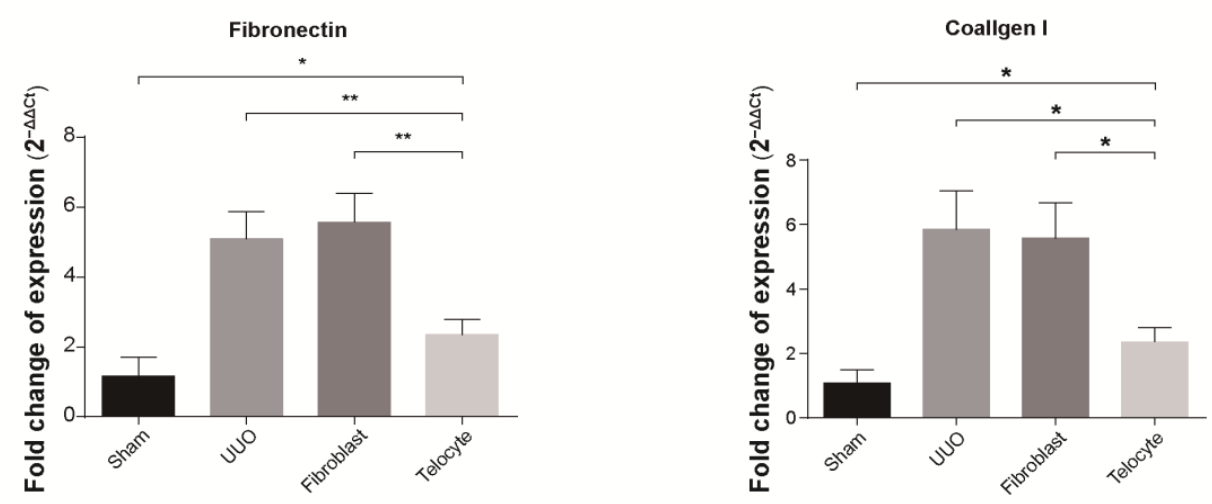

B
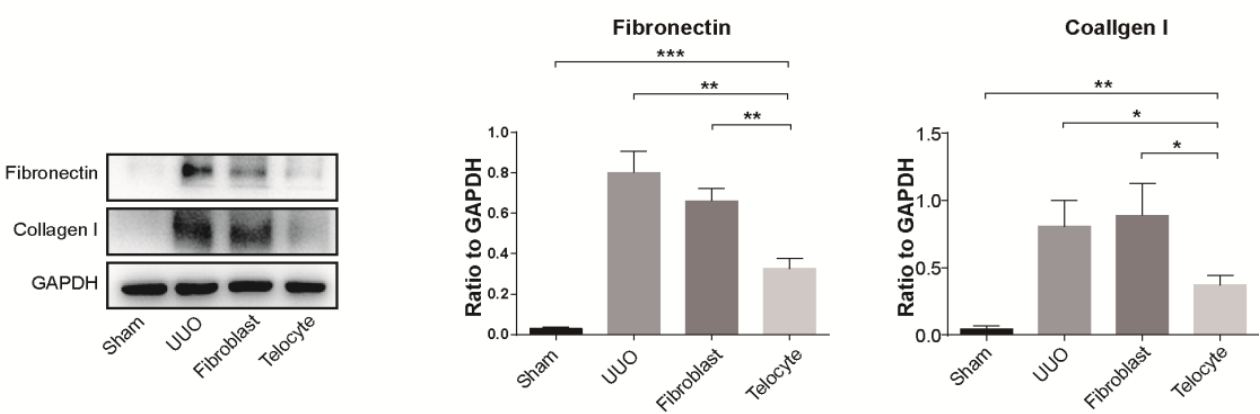

C
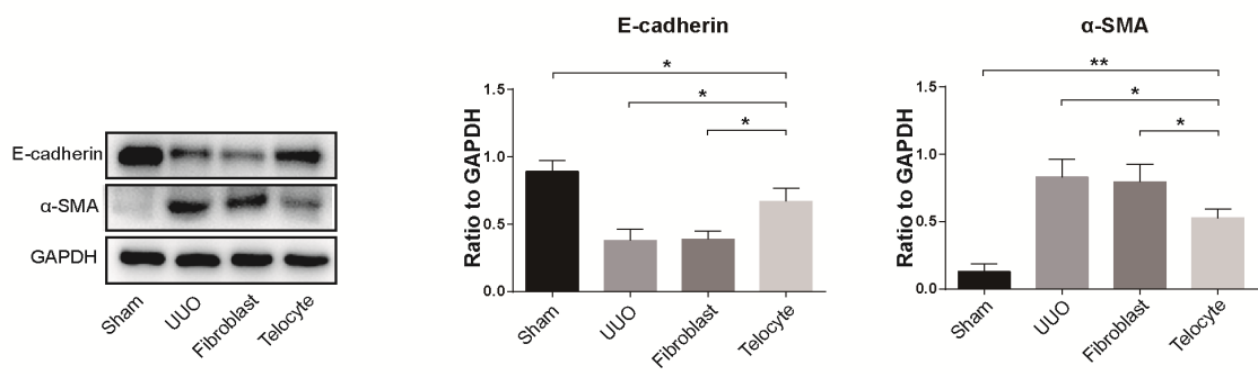

Fig. 3. Transplantation of telocytes inhibits ECM deposition and EMT in kidney tissue. (A) Fold changes of the mRNA expression levels of fibronectin and collagen I in renal tissue of all groups. (B) Representative western blot images of fibronectin and collagen I in all groups and the ratio of fibronectin and collagen I to GAPDH. (C) Representative western blot images of E-cadherin and $\alpha$-SMA in all groups and the ratio of E-cadherin and $\alpha$-SMA to GAPDH. Data are expressed as the mean $\pm \mathrm{SD}$. ${ }^{*} \mathrm{P}<0.05,{ }^{* *} \mathrm{P}<0.01,{ }^{* * *} \mathrm{P}<0.001$.

that telocyte transplantation inhibited the downstream signal transduction of the TGF- $\beta 1$ pathway. The PI3K/AKT and ERK/MAPK signaling pathways were also explored; however, there was no significant difference in the levels of p-Akt and p-ERK1/2 among the UUO, fibroblast, and telocyte groups (see online suppl. material, Suppl. Fig. 3).

\section{HGF expression is significantly higher in the telocyte group}

To elucidate the possible mechanism involved in the inhibition of the TGF- $\beta 1$ signaling pathway, some growth factors related to renal fibrogenesis were detected. As shown in Fig. 6A, the mRNA levels of PDGF, CTGF, EGF, and IGF-1 were significantly higher in the UUO group, but neither fibroblast nor telocyte treatment was able to cause a significant difference in their levels compared with the UUO group. Moreover, no significant difference was detected in the mRNA levels of BMP7 and HO-1 among the UUO, fibroblast, and telocyte groups. As 


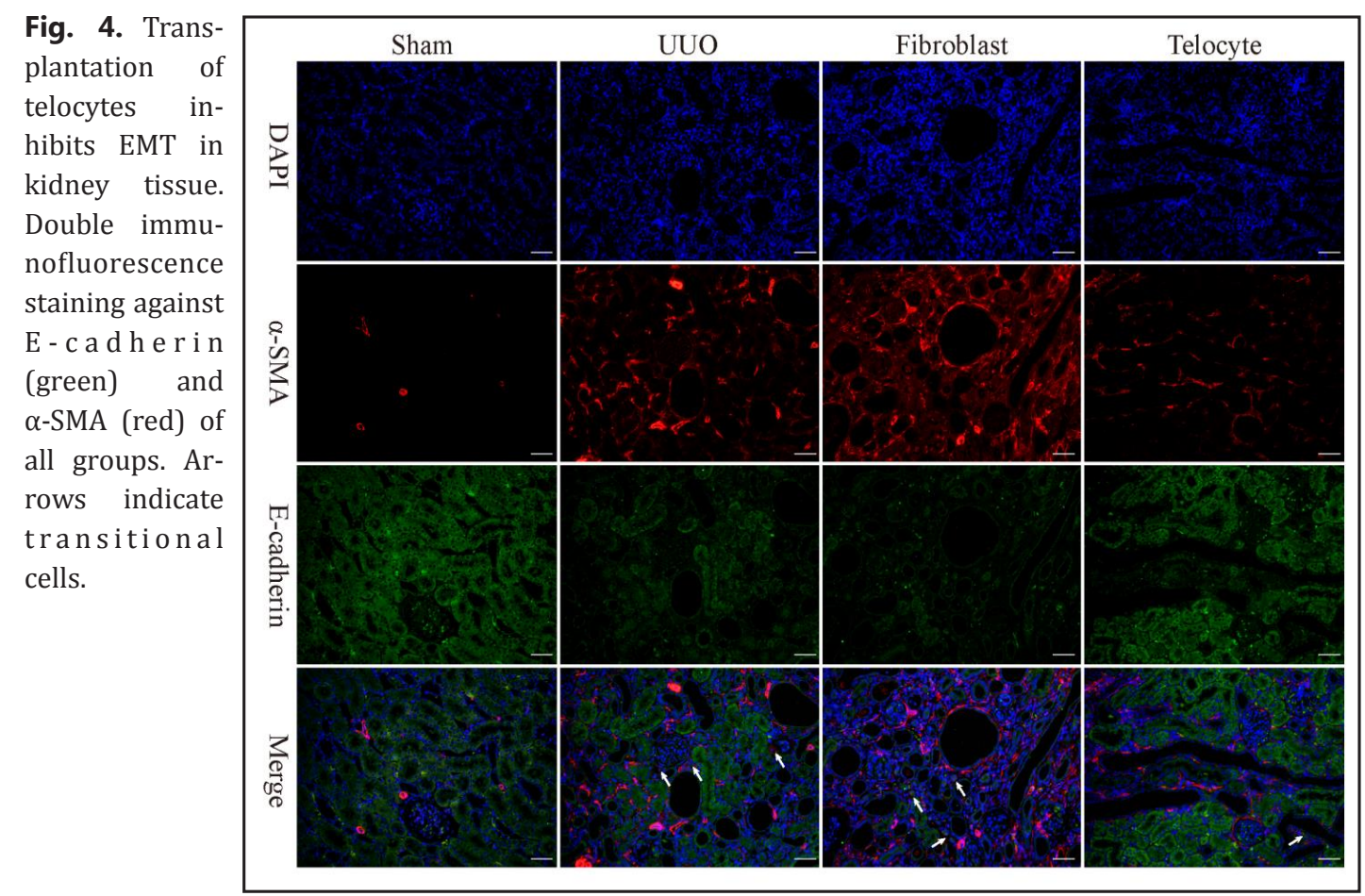

shown in Fig. 6B, the mRNA levels of HGF were high in the sham group, but significantly lower in both the UUO and fibroblast groups. Treatment with telocytes was able to enhance its expression. To confirm this result further, western blot analysis was performed to detect the expression of HGF. Besides, to verify whether TC transplantation allows the reconstruction of the TC interstitial network in the kidneys of UUO rats, we detected CD34 staining using immunohistochemistry and found that compared with sham group, lower expression of CD34 was detected in UUO group. Treatment with fibroblasts or TCs showed no obvious improvement in the expression of CD34 in interstitial area (see online suppl. material, Suppl. Fig. 4).

\section{Blockage of HGF aggravates renal fibrosis}

To investigate further the potential mechanism involved in the telocyte-mediated attenuation of renal fibrosis, western blot analysis was performed targeting the TGF- $\beta 1$ signaling pathway and renal fibrosis indicators after blocking HGF with an anti-HGF antibody. As shown in Fig. 7A, compared with the telocyte group, HGF expression was significantly lower in the anti-HGF antibody group, suggesting that treatment with the anti-HGF antibody successfully blocked HGF expression. Moreover, blocking HGF significantly increased the phosphorylation of Smad2/3 compared with the telocyte group, suggesting that blockage of HGF enhanced TGF- $\beta 1$ signal pathway transduction. These changes were also accompanied by the enhanced expression of fibronectin, collagen I, and $\alpha$-SMA as well as the decreased expression of E-cadherin, suggesting that blockage of HGF aggravated renal fibrosis.

\section{HGF is not secreted directly by telocytes}

To investigate whether the elevated expression of HGF was attributed to its secretion from telocytes, HGF mRNA levels were detected in fibroblasts and telocytes under two conditions. The mRNA levels of HGF were significantly lower in telocytes compared with fibroblasts under culture conditions. To explore whether HGF levels were affected by fibrotic conditions, a TGF- $\beta 1$-induced renal fibrosis model was used. Surprisingly, even under TGF$\beta 1$-induced fibrotic conditions, HGF mRNA levels were higher in fibroblasts than in telocytes, suggesting that the elevated levels of HGF within the system were not secreted directly by 


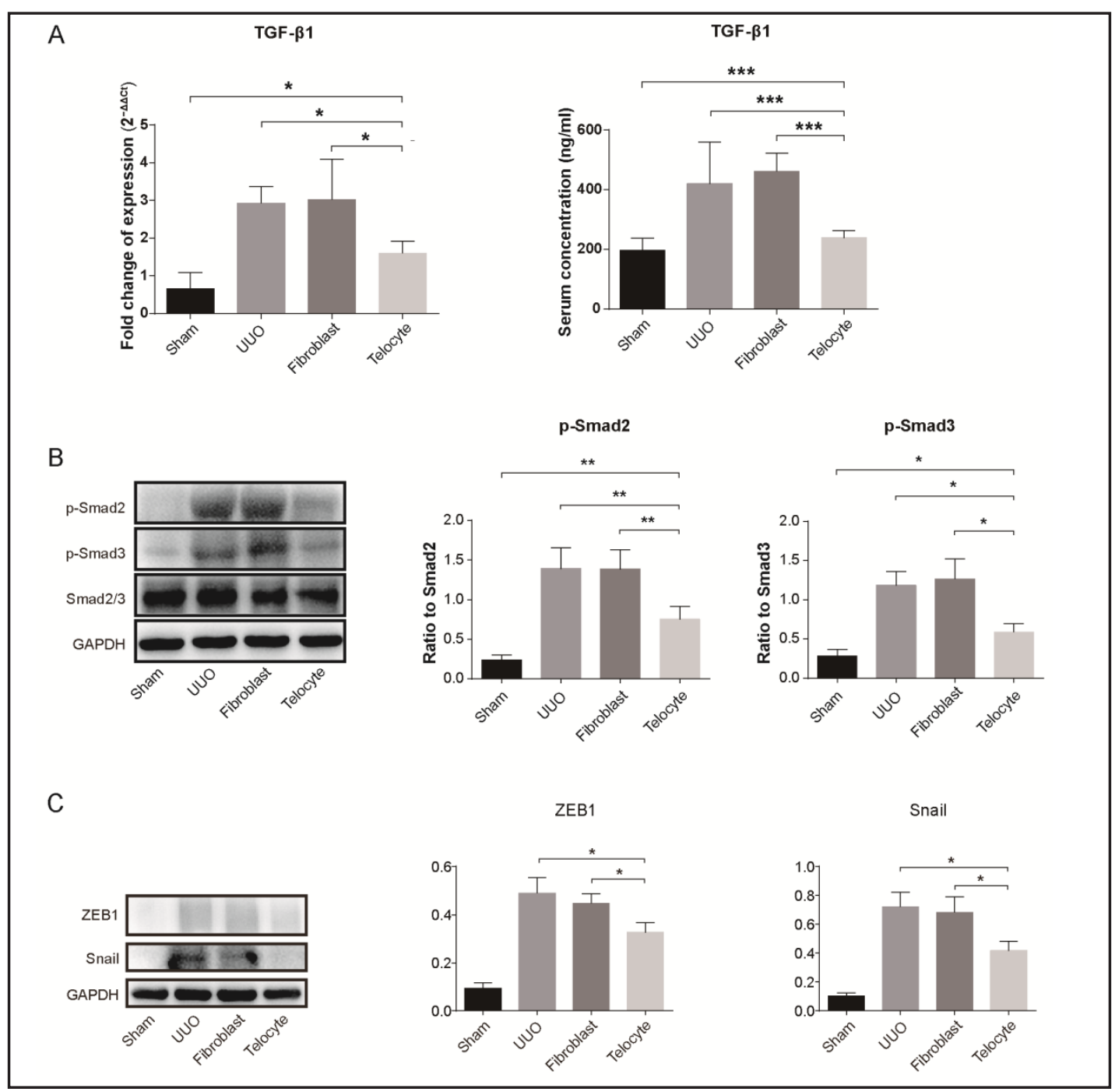

Fig. 5. Transplantation of telocytes inhibited TGF- $\beta 1$ synthesis and its downstream signaling pathway in kidney tissue. (A) Fold change of TGF- $\beta 1$ mRNA levels in renal tissue and quantification of serum TGF- $\beta 1$ levels in all groups. (B) Representative image of p-Smad2/3 and Smad2/3 levels in renal tissue and the ratio of p-Smad2/3 to Smad2/3 in all groups. (C) Representative western blot images of ZEB1 and Snail expression in renal tissue and the ratio of ZEB1 and Snail to GAPDH in all groups. Data are expressed as the mean $\pm \mathrm{SD} .{ }^{*} \mathrm{P}<0.05,{ }^{* *} \mathrm{P}<0.01,{ }^{* * *} \mathrm{P}<0.001$.

telocytes (Fig. 7B). Furthermore, western blot analysis revealed that compared with control group, the expression of E-cadherin was obviously lower, while $\alpha$-SMA expression was higher in the TGF- $\beta 1$ group. Co-culture with fibroblasts or telocytes did not alter the expression of these proteins, implying that telocytes have no effect on TGF- $\beta 1$-induced renal fibrosis in vitro (Fig. 7C).

\section{Discussion}

In this study, we found that the transplantation of telocytes inhibited ECM production and attenuated renal fibrosis in rats with UUO. The underlying mechanism may be mediated by the indirect secretion of HGF and inhibition of TGF- $\beta 1$ production and its downstream signaling pathway (Fig. 8). 

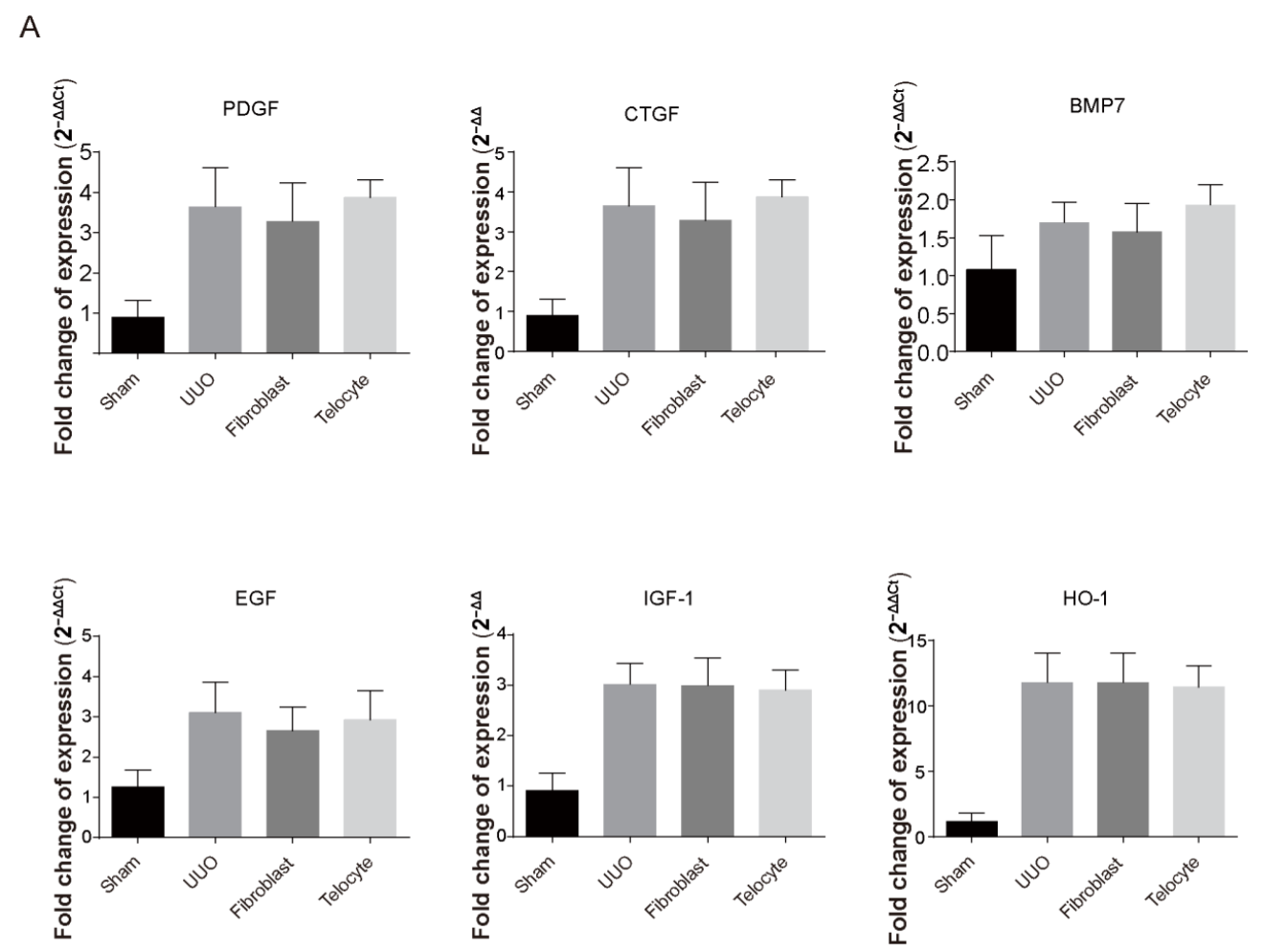

B
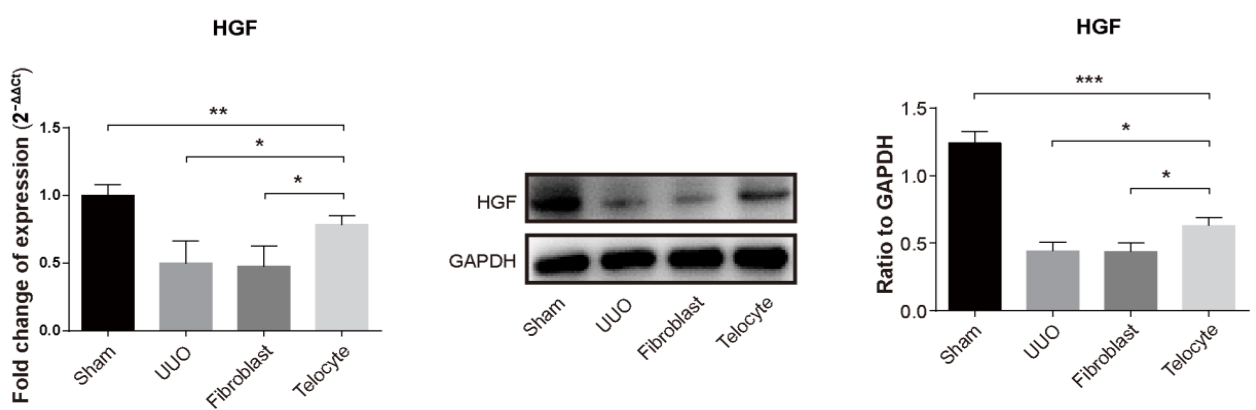

Fig. 6. HGF expression is significantly increased in the telocyte group. (A) Fold change of the mRNA levels of PDGF, CTGF, BMP7, EGF, IGF-1, and HO-1 in renal tissue in all groups. (B) Fold change of HGF mRNA levels, representative image of HGF expression, and the ratio of HGF to GAPDH in all groups. Data are expressed as the mean $\pm \mathrm{SD} .{ }^{*} \mathrm{P}<0.05,{ }^{* *} \mathrm{P}<0.01,{ }^{* * *} \mathrm{P}<0.001$.

Telocytes were first identified in the renal cortex by our team in 2012 [16], and the biological function of these cells in the kidney was subsequently explored. Li et al. of our laboratory found that transplantation of renal telocytes protects against renal ischemia reperfusion injury in rats, and the mechanism might be mediated by paracrine growth factors, including HGF, PDGF, IGF-1, and EGF, instead of inhibition of inflammation [22]. However, other potential biological functions of telocytes have not been explored, driving us to investigate the biological effect of these cells on renal fibrosis.

Renal fibrosis is the final consequence of renal disease, resulting in end-stage renal failure via the destruction of the kidney parenchyma and progression of renal malfunctions [30]. The normal overexpression and pathological accumulation of ECM components, such as fibronectin and collagen I, result in ECM deposition [31], thereby decreasing the glomerular infiltration rate, deteriorating renal function, and eventually leading to renal failure. In 


\section{A}
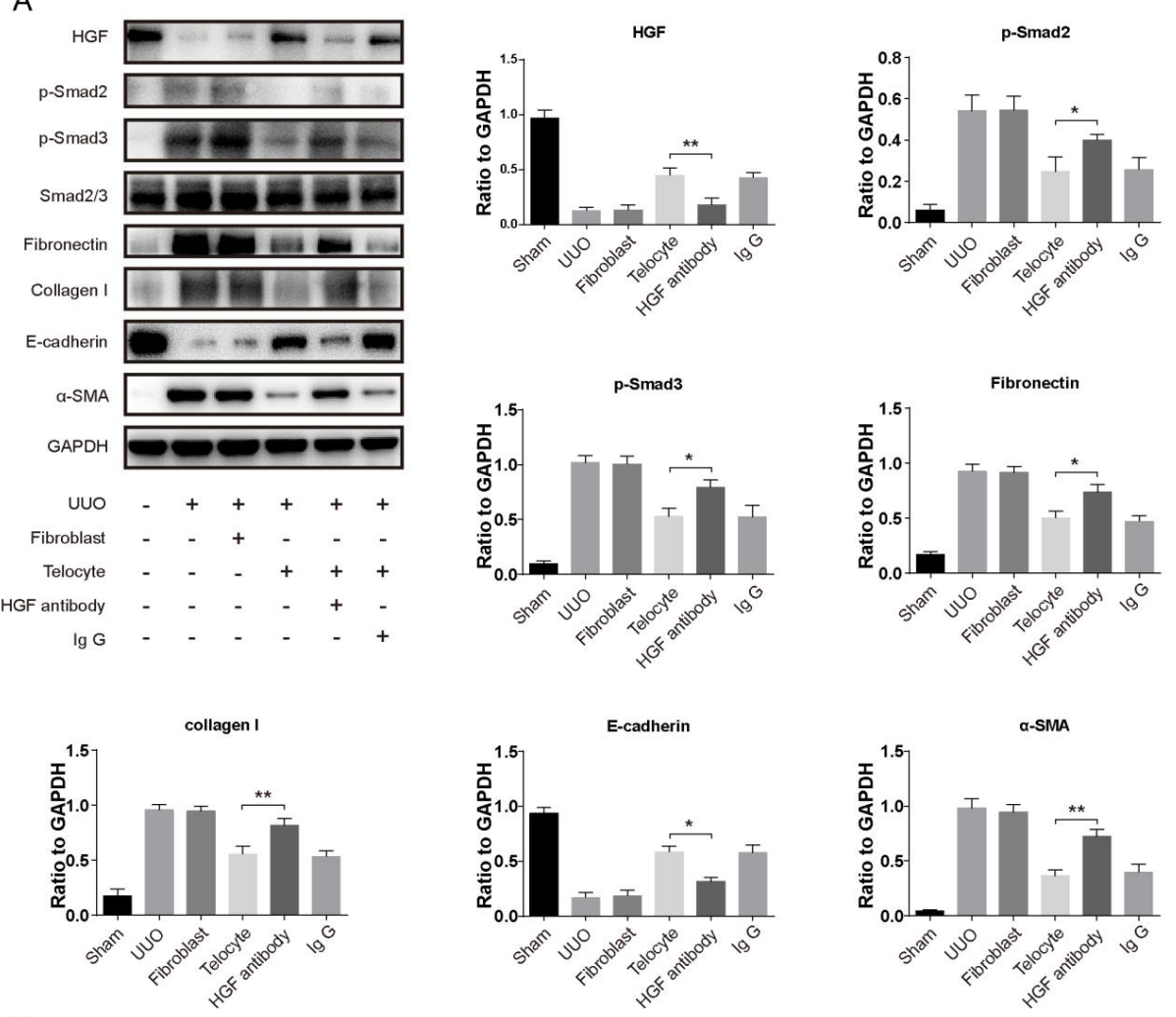

B

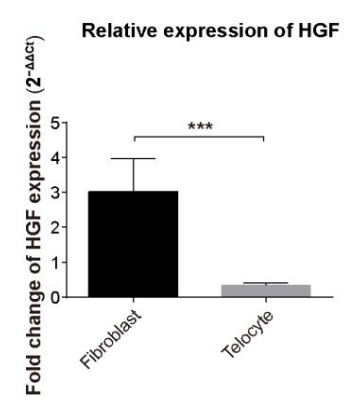

C
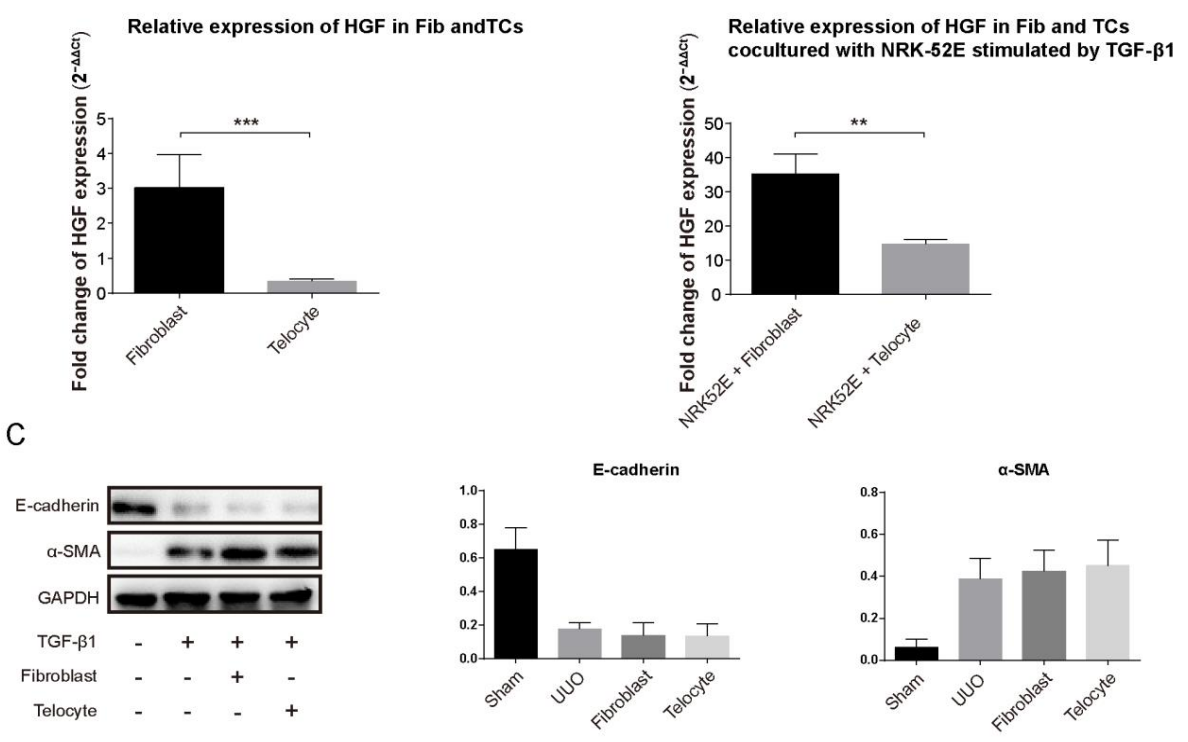

Fig. 7. Blockage of HGF aggravates renal fibrosis, while HGF is not secreted directly by telocytes. (A) Representative image of HGF, p-Smad2/3, Smad2/3, fibronectin, collagen I, E-cadherin, and $\alpha$-SMA expression in renal tissue, the ratio of HGF, fibronectin, collagen I, E-cadherin, and $\alpha$-SMA to GAPDH, and the ratio of p-Smad2/3 to Smad2/3. (B) Fold change of HGF in fibroblasts and telocytes; fold change of HGF in fibroblasts and telocytes co-cultured with NRK-52E cells stimulated by TGF- $\beta 1$, respectively. (C) Representative western blot images of E-cadherin and $\alpha$-SMA expression in renal tissues and the ratio of E-cadherin and $\alpha$-SMA to GAPDH in all groups. Data are expressed as the mean $\pm \mathrm{SD}$. ${ }^{*} \mathrm{P}<0.05,{ }^{* *} \mathrm{P}<0.01$, *** $\mathrm{P}<0.001$. 


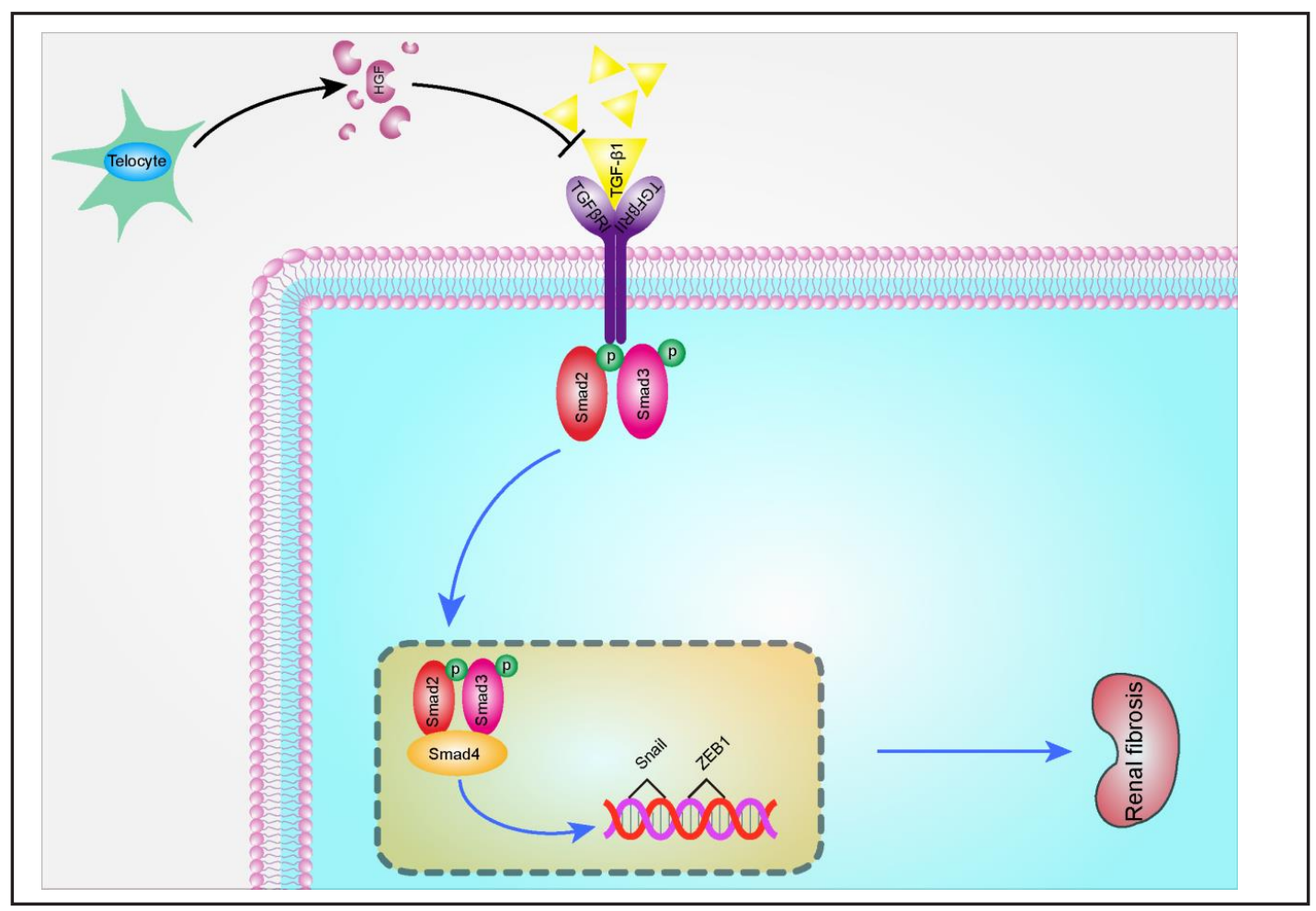

Fig. 8. Schematic diagram of the action of telocytes on the attenuation of renal fibrosis. The binding of TGF- $\beta 1$ and its receptor triggers the phosphorylation of Smad2 and Smad3. Once combined with Smad4, the Smad complex is formed. The complex is then transferred to the nucleus to regulate target gene transcription, such as Snail and ZEB1, resulting in renal fibrosis. Telocytes may indirectly promote the secretion of HGF, decreasing the level of TGF- $\beta 1$, thus attenuating renal fibrosis.

our study, we demonstrated that fibronectin and collagen I are significantly decreased by treatment with telocytes at both the protein and gene levels.

EMT, defined as the loss of epithelial characteristics and the acquisition of a mesenchymal phenotype, plays a vital role in the progression of fibrosis in the kidney [32]. During the process of EMT, tubular epithelial cells lose their phenotype (such as E-cadherin) and transform into fibroblasts/myofibroblasts, gaining a mesenchymal phenotype (such as $\alpha$-SMA) [33]. The enhanced conversion of renal tubular epithelial cells into fibroblasts/myofibroblasts leads to the disruption of polarized renal tubular epithelial layers and increase of fibrotic scar formation [32]. In the present study, we demonstrated that the expression of the epithelial marker E-cadherin and mesenchymal marker $\alpha$-SMA are reversed by telocytes treatment compared with the UUO and fibroblast treatment groups.

The TGF- $\beta$ superfamily contains various highly pleiotropic molecules, including activin, inhibin, bone morphogenic protein (BMP), and TGF- $\beta$ [34]. Among them, TGF- $\beta$ has been considered a key mediator of renal fibrosis with three subtypes, namely, TGF- $\beta 1$, TGF- $\beta 2$, and TGF- $\beta 3[35,36]$. TGF- $\beta 1$ is the most plentiful mediator and can be secreted by all cells in the kidney. After being secreted, TGF- $\beta 1$ binds to latency-associated peptide (LAP), forming latent TGF- $\beta 1$, which subsequently binds to latent TGF- $\beta$-binding protein (LTBP) in target tissue to form a complex. When this complex is exposed to multiple stimulating molecules, such as reactive oxygen and plasmin, TGF- $\beta 1$ is released from LAP and LTBP and turns into active TGF- $\beta 1[37,38]$. Previous studies indicated that TGF- $\beta 1$ exerts its biological functions in various manners, including the induction of ECM production [39], induction of cell loss through apoptosis [36], and direct induction of the transition of tubular epithelial cells to myofibroblasts $[4,39]$. In the present study, we found that the transplantation of telocytes in rats significantly decreased TGF- $\beta 1$ at both the protein and gene levels.

\section{KARGER}


The TGF- $\beta 1 /$ Smads signaling pathway is one of the most important pathways involved in renal fibrosis. The binding of TGF- $\beta 1$ and its receptor triggers the phosphorylation of Smad2 and Smad3. Once combined with Smad4, the Smad complex is formed. The complex is then transferred to the nucleus to regulate target gene transcription, including Snail and ZEB1, and thus resulting in renal fibrosis $[4,40,41]$. In the present study, we detected significantly increased phosphorylation of Smad2 and Smad3 and the expression of Snail and ZEB1 in the UUO group; while telocyte treatment was able to decrease the phosphorylation and expression of these proteins, fibroblast treatment did not result in a significant change. The PI3K/AKT and ERK/MAPK signaling pathways have also been considered to participate in renal fibrosis, yet the findings of this study indicate that there was no significant difference in the levels of p-Akt and p-ERK1/2 among the UUO, fibroblast, and telocyte groups.

HGF, EGF, PDGF, CTGF, BMP7, IGF-1, and HO-1 have been commonly recognized as cytokines that closely correlate with the occurrence of renal fibrosis [42-49]. EGF, PDGF, CTGF, and IGF-1 contribute to the progression of renal fibrosis [43-45], while HGF, BMP7, and HO- 1 attenuate renal fibrosis by counteracting the effect of TGF- $\beta[42,47,50]$. In the present study, we found no significant difference among the UUO, fibroblast-treated, and telocyte-treated groups in terms of EGF, PDGF, CTGF, BMP7, IGF-1, and HO-1. In contrast, HGF was significantly elevated at both the protein and gene levels compared with the UUO and fibroblast groups, suggesting that HGF may be secreted as a response to elevated TGF- $\beta 1$ levels, thus attenuating renal fibrosis.

To investigate the effect of HGF blockage on the TGF- $\beta 1$ signaling pathway and renal fibrosis, we examined the TGF- $\beta 1 /$ Smads pathway and indicators of renal fibrosis after using an anti-HGF antibody. We found that the application of the anti-HGF antibody clearly decreased HGF expression. Moreover, decreasing the expression of HGF significantly enhanced TGF- $\beta 1 /$ Smads pathway transduction. Enhancement of TGF- $\beta 1 /$ Smads pathway transduction increased the expression of fibronectin, collagen I, and $\alpha$-SMA and decreased the expression of E-cadherin. These results indicate that telocytes may attenuate renal fibrosis through the secretion of HGF.

We also examined the mRNA levels of HGF in fibroblasts and telocytes in vitro. The mRNA levels of HGF were significantly higher in fibroblasts than in telocytes. A TGF- $\beta 1$ induced renal fibrosis model was examined to explore whether HGF secretion was affected by pathological conditions, yet a similar result was observed. Besides, in the TGF- $\beta 1$-induced renal fibrosis model, no significant difference was observed in the expression of E-cadherin and $\alpha$-SMA among the TGF- $\beta 1$, fibroblast, and telocyte groups, suggesting that telocytes might promote HGF secretion in an indirect manner. This is consistent with a previous study, in which HGF was significantly elevated in a telocyte-treated ischemia reperfusion injury model to act as a vital paracrine regulator and protect against apoptosis [22].

\section{Conclusion}

This study revealed that telocytes attenuate UUO-induced renal fibrosis in rats. The underlying mechanism may involve the indirect enhancement of HGF by telocytes and subsequent decrease of TGF- $\beta 1$ and neutralization of its downstream effect.

\section{Acknowledgements}

This study was supported by the National Natural Science Foundation of China (grant nos. 81770747,81500568 and 81500570 ).

\section{Disclosure Statement}

The authors declare no competing interests. 


\section{Cellular Physiology Cell Physiol Biochem 2018;46:2056-2071 and Biochemistry \begin{tabular}{l|l} 
DOI: 10.1159/000489445 & $\begin{array}{l}\text { C } 2018 \text { The Author(s). Published by S. Karger AG, Basel } \\
\text { www.karger.com/cpb }\end{array}$
\end{tabular}}

Zheng et al.: Telocytes Attenuates Renal Fibrosis

\section{References}

1 Wynn TA: Common and unique mechanisms regulate fibrosis in various fibroproliferative diseases. J Clin Invest 2007;117:524-529.

- Guo Y, Li Z, Ding R, Li H, Zhang L, Yuan W, Wang Y: Parathyroid hormone induces epithelial-to-mesenchymal transition via the Wnt/beta-catenin signaling pathway in human renal proximal tubular cells. Int J Clin Exp Pathol 2014;7:5978-5987.

3 Wei C, Li L, Menon MC, Zhang W, Fu J, Kidd B, Keung KL, Woytovich C, Greene I, Xiao W, Salem F, Yi Z, He JC, Dudley JT, Murphy B: Genomic Analysis of Kidney Allograft Injury Identifies Hematopoietic Cell Kinase as a Key Driver of Renal Fibrosis. J Am Soc Nephrol 2016;28:1385-1393

4 Lan HY: Diverse roles of TGF-beta/Smads in renal fibrosis and inflammation. Int J Biol Sci 2011;7:10561067.

-5 Zhou X, Sun X, Gong X, Yang Y, Chen C, Shan G, Yao Q: Astragaloside IV from Astragalus membranaceus ameliorates renal interstitial fibrosis by inhibiting inflammation via TLR4/NF-small ka, CyrillicB in vivo and in vitro. Int Immunopharmacol 2016;42:18-24.

6 Remuzzi G, Bertani T: Pathophysiology of progressive nephropathies. N Engl J Med 1998;339:1448-1456.

7 Eddy AA: Molecular basis of renal fibrosis. Pediatr Nephrol 2000;15:290-301.

8 Popescu LM, Manole CG, Gherghiceanu M, Ardelean A, Nicolescu MI, Hinescu ME, Kostin S: Telocytes in human epicardium. J Cell Mol Med 2010;14:2085-2093.

-9 Gherghiceanu M, Manole CG, Popescu LM: Telocytes in endocardium: electron microscope evidence. J Cell Mol Med 2010;14:2330-2334.

10 Suciu L, Nicolescu MI, Popescu LM: Cardiac telocytes: serial dynamic images in cell culture. J Cell Mol Med 2010;14:2687-2692.

11 Zheng Y, Li H, Manole CG, Sun A, Ge J, Wang X: Telocytes in trachea and lungs. J Cell Mol Med 2011;15:22622268.

-12 Rusu MC, Nicolescu MI, Jianu AM, Lighezan R, Mănoiu VS, Păduraru D: Esophageal telocytes and hybrid morphologies. Cell Biol Int 2012;36:1079-1088.

13 Nicolescu MI, Popescu LM: Telocytes in the interstitium of human exocrine pancreas: ultrastructural evidence. Pancreas 2012;41:949-956.

14 Cantarero Carmona I, Luesma Bartolome MJ, Junquera Escribano C: Identification of telocytes in the lamina propria of rat duodenum: transmission electron microscopy. J Cell Mol Med 2011;15:26-30.

15 Cretoiu D, Cretoiu SM, Simionescu AA, Popescu LM: Telocytes, a distinct type of cell among the stromal cells present in the lamina propria of jejunum. Histol Histopathol 2012;27:1067-1078.

16 Qi G, Lin M, Xu M, Manole CG, Wang X, Zhu T: Telocytes in the human kidney cortex. J Cell Mol Med 2012;16:3116-3122.

17 Vannucchi MG, Traini C, Manetti M, Ibba-Manneschi L, Faussone-Pellegrini MS: Telocytes express PDGFRalpha in the human gastrointestinal tract. J Cell Mol Med 2013;17:1099-1108.

18 Ibba-Manneschi L, Rosa I, Manetti M: Telocyte implications in human pathology: An overview. Semin Cell Dev Biol 2016;55:62-69.

19 Cismasiu VB, Radu E, Popescu LM: miR-193 expression differentiates telocytes from other stromal cells. J Cell Mol Med 2011;15:1071-1074.

20 Zheng Y, Zhang M, Qian M, Wang L, Cismasiu VB, Bai C, Popescu LM, Wang X: Genetic comparison of mouse lung telocytes with mesenchymal stem cells and fibroblasts. J Cell Mol Med 2013;17:567-77.

-21 Zheng Y, Cretoiu D, Yan G, Cretoiu SM, Popescu LM, Wang X: Comparative proteomic analysis of human lung telocytes with fibroblasts. J Cell Mol Med 2014;18:568-589.

22 Li L, Lin M, Li L, Wang R, Zhang C, Qi G, Xu M, Rong R, Zhu T: Renal telocytes contribute to the repair of ischemically injured renal tubules. J Cell Mol Med 2014;18:1144-1156.

23 Manetti M, Guiducci S, Ruffo M, Rosa I, Faussone-Pellegrini MS, Matucci-Cerinic M, Ibba-Manneschi L: Evidence for progressive reduction and loss of telocytes in the dermal cellular network of systemic sclerosis. J Cell Mol Med 2013;17:482-496.

24 Manetti M, Rosa I, Messerini L, Ibba-Manneschi L: Telocytes are reduced during fibrotic remodelling of the colonic wall in ulcerative colitis. J Cell Mol Med 2015;19:62-73.

25 Milia AF, Ruffo M, Manetti M, Rosa I, Conte D, Fazi M, Messerini L, Ibba-Manneschi L: Telocytes in Crohn's disease. J Cell Mol Med 2013;17:1525-1536. 


\section{Cellular Physiology Cell Physiol Biochem 2018;46:2056-2071 \begin{tabular}{ll|l} 
and Biochemistry Published online: NlVy 03, 2018 & $\begin{array}{l}\text { (c) 2018 The Author(s). Published by S. Karger AG, Basel } \\
\text { www.karger.com/cpb }\end{array}$ \\
\hline
\end{tabular}

-26 Zhaofu L, Dongqing C: Cardiac Telocytes in Regeneration of Myocardium After Myocardial Infarction. Adv Exp Med Biol 2016;913:229-239.

27 Fu S, Wang F, Cao Y, Huang Q Xiao J, Yang C, Popescu LM: Telocytes in human liver fibrosis. J Cell Mol Med 2015;19:676-683.

-28 Meng J, Li L, Zhao Y, Zhou Z, Zhang M, Li D, Zhang CY, Zen K, Liu Z: MicroRNA-196a/b Mitigate Renal Fibrosis by Targeting TGF-beta Receptor 2 J Am Soc Nephrol 2016;27:3006-3021.

-29 Lin M, Li L, Li L, Pokhrel G, Qi G, Rong R1, Zhu T: The protective effect of baicalin against renal ischemiareperfusion injury through inhibition of inflammation and apoptosis. BMC Complement Altern Med DOI:10.1186/1472-6882-14-19.

-30 Eddy AA, Neilson EG: Chronic kidney disease progression. J Am Soc Nephrol 2006;17:2964-2966.

-31 Shen Y, Miao N, Xu J, Gan X, Xu D, Zhou L, Xue H, Zhang W, Lu L: Metformin Prevents Renal Fibrosis in Mice with Unilateral Ureteral Obstruction and Inhibits Ang II-Induced ECM Production in Renal Fibroblasts. Int J Mol Sci DOI:10.3390/ijms17020146

32 Yoshinaga T, Uwabe K, Naito S, Higashino K, Nakano T, Numata Y, Kihara A: AM251 Suppresses EpithelialMesenchymal Transition of Renal Tubular Epithelial Cells. PLoS One 2016;11:e0167848.

-33 Hung TW, Tsai JP, Lin SH, Lee CH, Hsieh YH, Chang HR: Pentraxin 3 Activates JNK Signaling and Regulates the Epithelial-To-Mesenchymal Transition in Renal Fibrosis. Cell Physiol Biochem 2016;40:1029-1038.

34 Massague J, Wotton D: Transcriptional control by the TGF-beta/Smad signaling system. EMBO J 2000;19:1745-1754.

-35 Vega G, Alarcon S, San Martin R: The cellular and signalling alterations conducted by TGF-beta contributing to renal fibrosis. Cytokine 2016;88:115-125.

-36 Meng XM, Nikolic-Paterson DJ, Lan HY: TGF-[beta]: the master regulator of fibrosis. Nat Rev Nephrol 2016;12:325-338.

-37 Lyons RM, Gentry LE, Purchio AF, Moses HL: Mechanism of activation of latent recombinant transforming growth factor beta 1 by plasmin. J Cell Biol 1990;110:1361-1367.

38 Meng XM, Nikolic-Paterson DJ, Lan HY: TGF-beta: the master regulator of fibrosis. Nat Rev Nephrol 2016;12:325-338.

-39 Lan HY: Tubular epithelial-myofibroblast transdifferentiation mechanisms in proximal tubule cells. Curr Opin Nephrol Hypertens 2003;12:25-29.

40 Sureshbabu A, Muhsin SA, Choi ME: TGF-beta Signaling in the Kidney: Pro-fibrotic and Protective Effects. Am J Physiol Renal Physiol DOI:10.1152/ajprenal.00365.

41 Guo L, Peng W, Tao J, Lan Z, Hei H, Tian L, Pan W, Wang L, Zhang X: Hydrogen Sulfide Inhibits Transforming Growth Factor-beta1-Induced EMT via Wnt/Catenin Pathway. PLoS One 2016;11:e0147018.

42 He D, Yang J, Wang X: Cordycepin inhibits renal interstitial myofibroblast activation probably by inducing hepatocyte growth factor expression. J Pharmacol Sci 2011;117:286-294.

-43 Chen J, Chen JK, Nagai K, Plieth D, Tan M, Lee TC, Threadgill DW, Neilson EG, Harris RC: EGFR signaling promotes TGFbeta-dependent renal fibrosis. J Am Soc Nephrol 2012;23:215-224.

44 Ostendorf T, Boor P, van Roeyen CR, Floege J: Platelet-derived growth factors (PDGFs) in glomerular and tubulointerstitial fibrosis. Kidney Int Suppl 2014;4:65-69.

45 Ren Y, Du C, Yan L, Wei J, Wu H, Shi Y, Duan H: CTGF siRNA ameliorates tubular cell apoptosis and tubulointerstitial fibrosis in obstructed mouse kidneys in a Sirt1-independent manner. Drug Des Devel Ther 2015;9:4155-4171.

46 Kok HM, Falke LL, Goldschmeding R, Nguyen TQ: Targeting CTGF, EGF and PDGF pathways to prevent progression of kidney disease. Nat Rev Nephrol 2014;10:700-711.

47 Massague J, Chen YG: Controlling TGF-beta signaling. Genes Dev 2000;14:627-644.

48 Meng XM, Chung AC, Lan HY: Role of the TGF-beta/BMP-7/Smad pathways in renal diseases. Clin Sci (Lond) 2013;124:243-254.

49 Lv S, Liu G, Sun A, Wang J, Cheng J, Wang W, Liu X, Nie H, Guan G: Mesenchymal stem cells ameliorate diabetic glomerular fibrosis in vivo and in vitro by inhibiting TGF-beta signalling via secretion of bone morphogenetic protein 7. Diab Vasc Dis Res 2014;11:251-261.

50 Yang J, Dai C, Liu Y; Hepatocyte growth factor suppresses renal interstitial myofibroblast activation and intercepts Smad signal transduction. Am J Pathol 2003;163:621-32. 